\title{
PENILE GROWTH WITH UNUSUAL HISTOPATHOLOGY: A CASE REPORT
}

\author{
SK. AMIRULISLAM ${ }^{1}$, MD. MONOWARUL ISLAM ${ }^{1}$, SHUVENDRA NATH NAG ${ }^{1}$, MD. SIDDIQUR RAHMAN ${ }^{1}$, \\ HOWLADER FAZLUL KARIMํ, MOHAMMAD MURAD CHOWDHURY ${ }^{1}$, FARIDA ARJUMAN², \\ PRANASHIS SAHA ${ }^{1}$
}

${ }^{1}$ Department of Uro-Oncology, NICRH, Mohakhali, Dhaka-1212, ${ }^{2}$ Department of Histopathology, NICRH, Mohakhali, Dhaka-1212

Bangladesh J. Urol. 2017; 20(2): 105-108

\section{Introduction}

Pseudoepitheliomatous hyperplasia $(\mathrm{PEH})$ is a benign condition characterized by hyperplasia of the epidermis and adnexal epithelium closely simulating squamous cell carcinoma(SCC)[1]. PEH is commonly mistaken for SCC. PEH may be present in a number of conditions characterized by prolonged inflammation and/or chronic infection, as well as in association with many cutaneous neoplasms[2]. Pseudoepitheliomatous hyperplasia of penis is an extremely rare pathology considered a distinct clinical entity by some, while others view it as a subset of verrucous carcinoma with premalignant potential[3,4]. PEH can be managed by surgical excision with adequate margin, hence it has to be

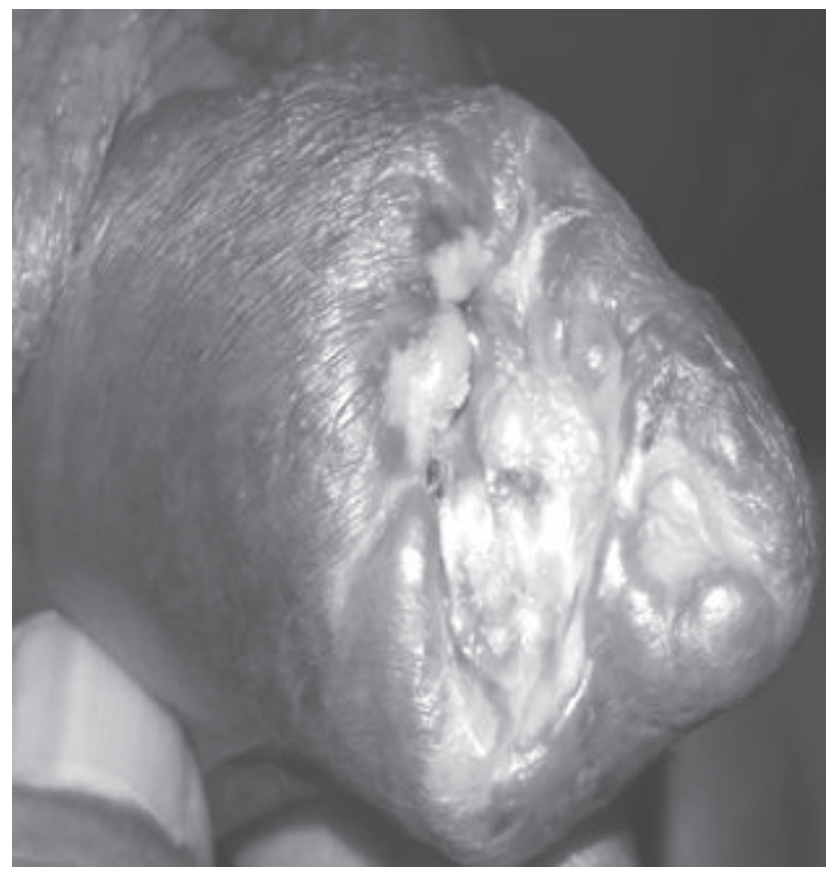

Figure $1 \mathrm{~A}$ : Penile growth before excision differentiated from other mimickers of SCC both benign and malignant ${ }^{1}$. Given the lack of data and sparse literature regarding this unusual entity, pseudoepitheliomatous hyperplasia continues to pose a challenging clinical problem with uncertain prognosis $[5,6]$. Here we present a 55 yrs old male with PEH presenting with growth involving glans \& distal penile shaft.

\section{Case Report}

A 55-year-old male patient presented with a growth over glans and distal penis since 6 months duration. It was initially small in size and gradually progressed to attain present size. It was not associated with itching or burning

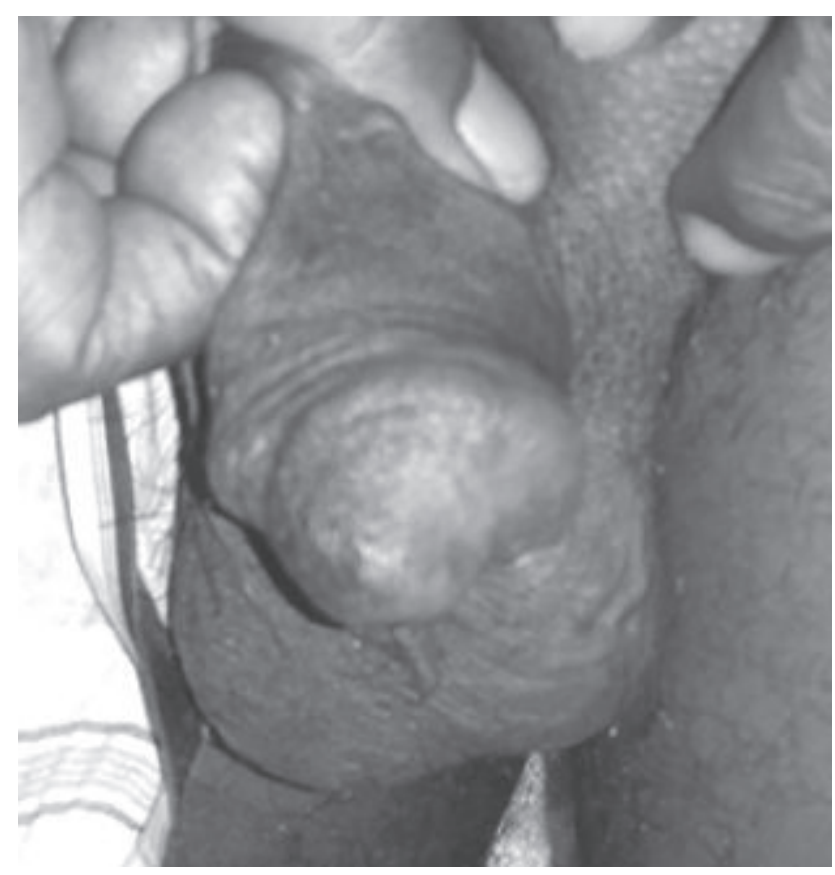

Figure 1B: After excision

Correspondences: Dr. Sk. Amirul Islam, Department of Uro-oncology, National Institute of Cancer Research and Hospital (NICRH), Mohakhali, Dhaka-1212. Email: amiruluro@yahoo.com 
sensation. Patient was non diabetic and there was no history suggestive of sexually transmitted infections. There was no history of bleeding either spontaneously or following minor trauma. Patient was circumcised. Clinical examination revealed there was an irregular growth with a non healing ulcer over glans \& distal penile shaft measured $2 \times 2 \mathrm{~cm}$, growth is hard \& surface nodular, there was discharge from the ulcer floor of the ulcer showing unhealthy granulation tissue with irregular margin. Inguinal lymph nodes were not enlarged. On blood investigations, complete blood count and blood glucose level were within normal limit. Serological tests for human immunodeficiency virus, hepatitis B and syphilis were negative. Incisional biopsy was performed under spinal anesthesia, which on histopathology revealed pseudoepitheliomatous hyperplasia,

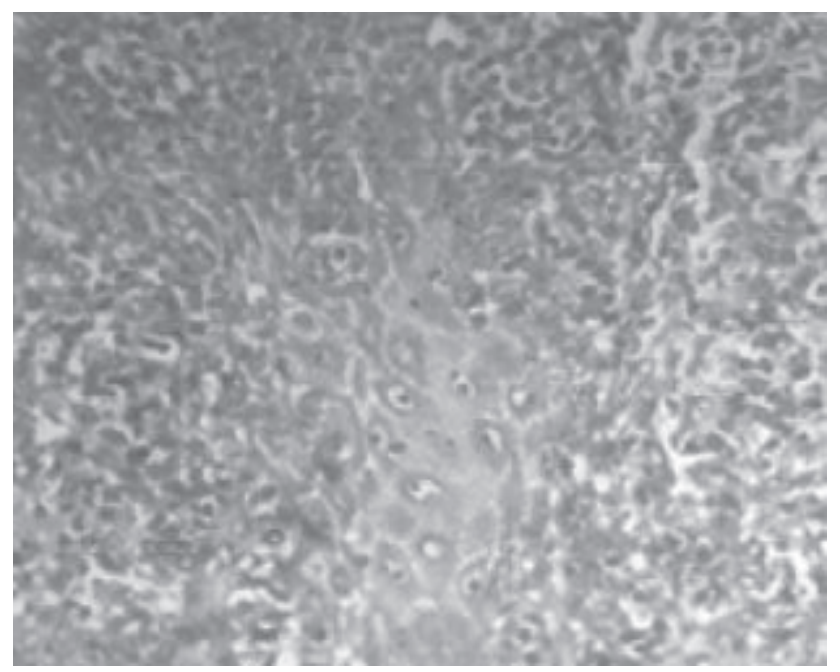

Figure 2A: Primary Histopathology Slide

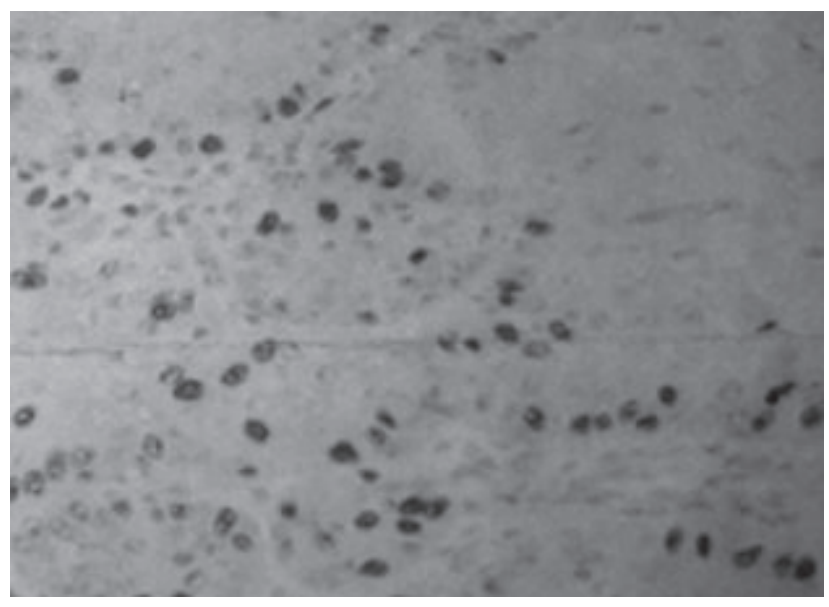

Figure2C: Immunohistochemistry acanthosis, elongation of rete ridges, and nonspecific dermal acute and chronic inflammatory cellular infiltrates [Fig-2A]. There was no cytological atypia, koilocytes or evidence of malignancy. We reviwed the slides for immunostaining by $\mathrm{p} 16, \mathrm{ki} 67$ and SMA was performed to differentiate from well differentiated squamous cell carcinoma and it confirms the PEH [Fig2B,C\&D]. On clinical suspicion and immunohistochemistry report we planned for wide local excision. Wide local excision of growth with penile reconstruction was done. No penectomy was performed. Subsequent histopathology demonstrated a benign pseudoepitheliomatous hyperplasia with clear margins (Fig-3A\& B). A follow up CT scan 4 weeks later demonstrate nothing abnormality. The patient will be closely observed with biannual physical examination for recurrence.

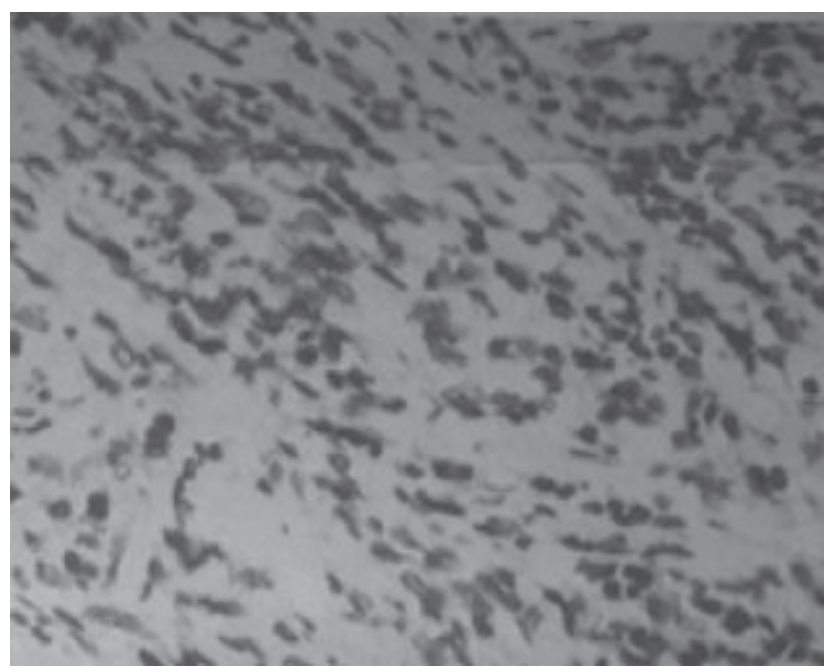

Figure 2B: Immunohistochemistry

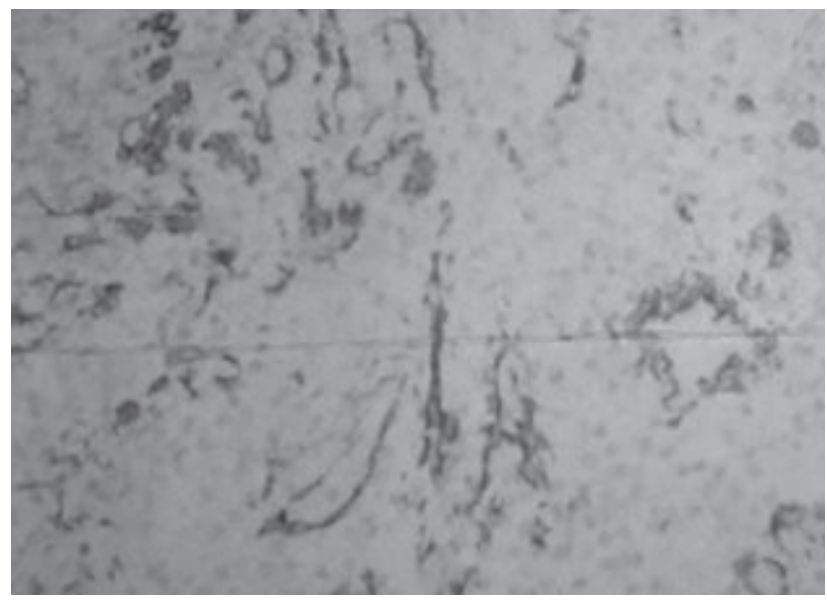

Figure2D: Immunohistochemistry 

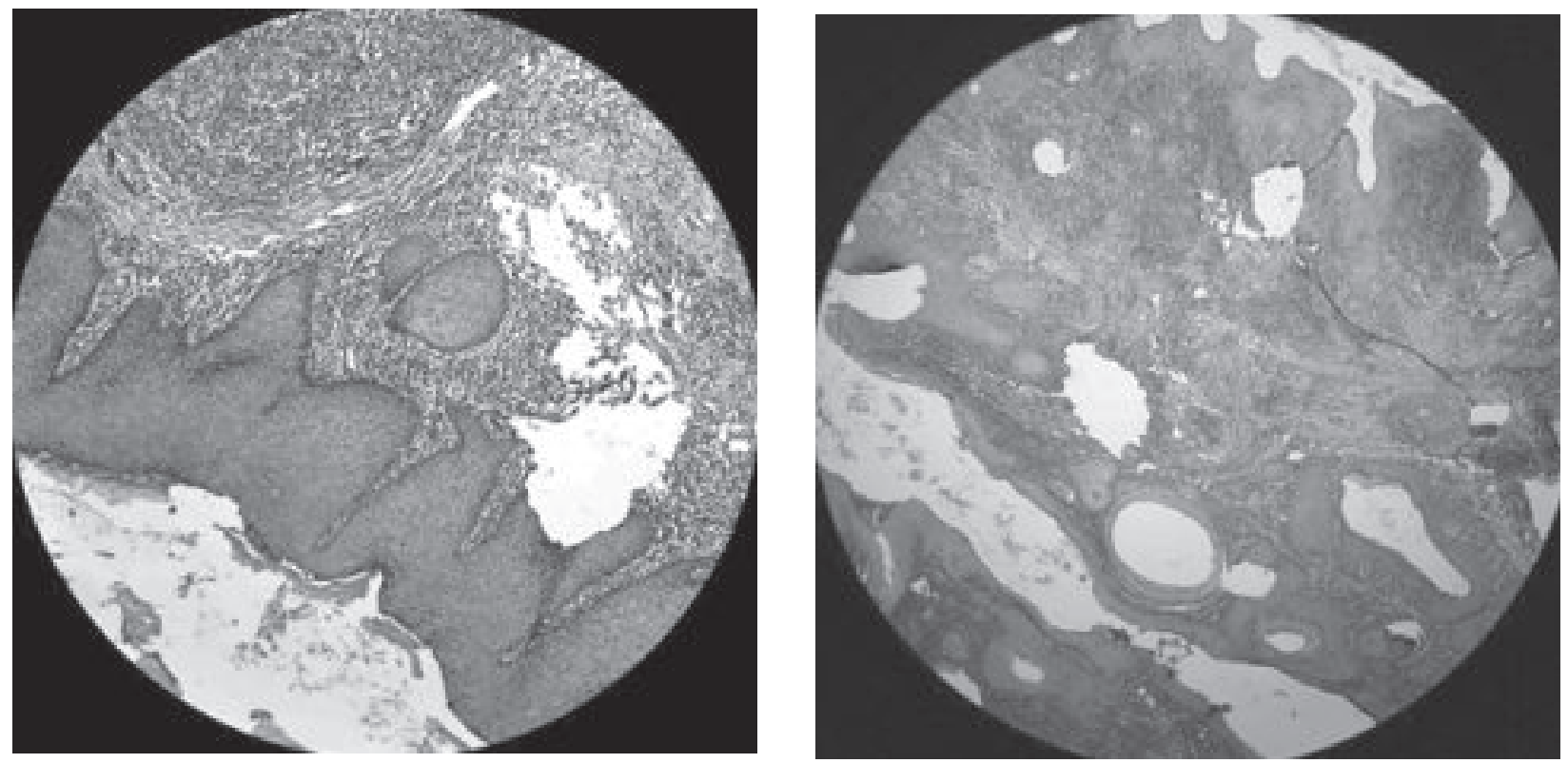

Figure 3A\& B: Section from penile growth show prominent acanthosis, elongation of rete ridges. Subepithelial tissue shows acute \& chronic inflammatory cell infiltration

\section{Discussion}

Literature revealed that Penile pseudoepitheliomatous hyperplasia is a rare histopathologic diagnosis affecting the glans penis, first described in 1961 as pseudoepitheliomatous keratotic and micaceous balanitis (PKMB) of Civatte in French literature ${ }^{3}$. It is a acquired penile disorder affecting glans penis of elderly men which is characterized by thick hyperkeratotic plaque which often leading to phimosis. Most patients are over the age of 60 and frequently have been circumcised for phimosis in adult life. It is often asymptomatic but may be associated with irritation or burning sensation ${ }^{7}$. While little is known about the risk factors or pathogenesis, this disease appears to be most closely associated with adult circumcision ${ }^{3}$. In this patient, neither of these typical findings or associations exists. Classical histopathological examination reveals hyperkeratosis, parakeratosis, prominent acanthosis, elongation of rete ridges. Subepithelial tissue shows acute \& chronic inflammatory cell infiltration. Some authors considered it as a variant of lichen sclerosis ${ }^{7}$. Regarding prognosis, a review of the literature by Perry et al in 2008 revealed a total of 14 case reports of PKMB. Five patients progressed to verrucous carcinoma, four progressed to squamous cell carcinoma, and one patient progressed to verrucous carcinoma and then subsequently to squamous cell carcinoma. The authors

concluded that PKMB has malignant potential if inadequately treated ${ }^{3}$. However, it's prognosis and risk of recurrence or progression to malignancy is uncertain. Our patient has been adequately treated with surgical excision with negative margin and we will continue to monitor him for clinical recurrence.

\section{Conclusion}

$\mathrm{PEH}$ is a benign condition which has to be differentiated from SCC, as the treatment and prognosis for the patient changes. Erroneous diagnosis of malignancy will lead to radical surgery and surgery-related morbidity. Though it is a benign condition it's risk of malignant transformation is uncertain. So long term follow up is needed for early detection of malignant transformation.

\section{References:}

1. Chakrabarti S, Chakrabarti PR, Agrawal D \& Somanath S. Pseudoepitheliomatoushyperplasia: A clinical entity mistaken for squamous cell carcinoma. J Cutan Aesthet Surg.2014 Oct-Dec; 7(4): 232-234.

2. Zayour M, Lazova R. Pseudoepitheliomatous hyperplasia: A review. Am J Dermatopathol.2011; 33: 112-126. 
3. Johnson N, Voznesensky M \& VerLee G. Large penile mass with unusual benign histopathology. Urology Case Reports. 2015; 3:155-156.

4. Bashir SJ, Grant JW, Burrows NP. Pseudoepitheliomatous, keratotic and micaceousbalanitis after penile squamous cell carcinoma. Clin Exp Dermatol. 2010 Oct;35(7):749-751.

5. Murthy P S, Kanak K, Raveendra L, Reddy P. Pseudoepitheliomatous, keratotic, and micaceousbalanitis. Indian J Dermatol.2010;55:190-91.
6. Perry D, Lynch PJ, Fazel N. Pseudoepitheliomatous, keratotic, and micaceousbalanitis: case report and review of the literature. Dermatol Nurs. 2008;20:117- 20.

7. Malkud S, Dyavannanavar V. Pseudoepitheliomatous, keratotic, and micaceousbalanitis: A case report. Journal of Clinical and Diagnostic Research. 2015 Oct; 9(10): WD01WD02. 